\title{
ON THE FAILURE OF THE FIRST PRINCIPLE OF SEPARATION FOR COANALYTIC SETS ${ }^{1}$
}

\author{
ASHOK MAITRA
}

ABSTRACT. In this note we present a new example of a pair of dis joint coanalytic sets which are not Borel separable, i.e., coanalytic sets $D$ and $H$ such that $D \cap H=\varnothing$ and such that there is no Borel set $E$ for which $D$ $\subseteq E$ and $E \cap H=\varnothing$.

1. There are in the literature several proofs of the existence of a pair of disjoint coanalytic sets which are not Borel separable [3, Pp. 220, 260, 263], [4, p. 25], [5]. In this note we present yet another proof. We show that Blackwell's construction in [1] of a Borel set which does not admit a Borel uniformization yields explicitly a pair of disjoint coanalytic sets which are not Borel separable.

2. First, we briefly recall Blackwell's construction. Let $U$ be the set of all finite sequences of positive integers of positive length. Let $X$ be the power-set of $U$. Identify $X$ with $2^{U}$ and endow $X$ with the product of discrete topologies, so that $X$ is a homeomorph of the Cantor set. With each $x \in X$, associate a game $G(x)$ between players $\alpha$ and $\beta$ as fol. lows: the players alternately choose positive integers, $\alpha$ choosing first, each choice being made with complete information about all previous choices. For any play $\omega=\left(n_{1}, n_{2}, \ldots\right)$, let $k(\omega)$ be the first $i$ such that $\left(n_{1}, n_{2}, \cdots, n_{i}\right) \notin x$, and let $k(\omega)=\infty$ if $\left(n_{1}, n_{2}, \cdots, n_{i}\right) \in x$ for all $i$. A play $\omega$ is a win for $\alpha$ in $G(x)$ just in case $k(\omega)$ is even, it is a win for $\beta$ if $k(\omega)$ is odd, and it is a draw if $k(\omega)=\infty$. In any game $G(x)$, the space $Y_{1}$ of strategies for $a$ can be identified with the set $N^{N}$ of (infinite) sequences of positive integers, which we equip with the product of discrete

Received by the editors August 15, 1973.

AMS (MOS)! subject classifications (1970). Primary 04A15, 54H05; Secondary $02 \mathrm{~K} 30$.

Key words and phrases. Analytic sets, coanalytic sets, Borel separable, first principle of separation.

${ }^{1}$ Research supported by NIH Research Grant No. GM-10525, National Institutes of Health, Public Health Service. 
topologies. A similar remark applies to the space $Y_{2}$ of strategies for $\beta$. Let $Y$ be the disjoint union of $Y_{1}$ and $Y_{2}$ and give $Y$ the union topology, so that $Y$ is a homeomorph of $N^{N}$. Finally, let $B_{1}$ be the set of $(x, y) \epsilon$ $X \times Y_{1}$ such that $y$ ensures $\alpha$ at least a draw in $G(x)$, and let $B_{2}$ be the set of $(x, y) \in X \times Y_{2}$ such that $y$ ensures $\beta$ at least a draw in $G(x)$.

Then, Blackwell has proved that

(i) $B_{1}, B_{2}$ are Borel subsets of $X \times Y$ (indeed, $B_{1}, B_{2}$ are closed subsets of $X \times Y$ );

(ii) $\pi\left(B_{1} \cup B_{2}\right)=X$, where $\pi$ denotes projection of $X \times Y$ to $X$;

(iii) with each $x \in X$, it is possible to associate $x^{\prime} \in X$ and an ordered pair $\left(A_{1}, A_{2}\right)$ of nonempty analytic subsets of $X$ in such a way that

(a) $G\left(x^{\prime}\right)$ is a win for $\alpha$ if $x \in A_{1}-A_{2}$,

(b) $G\left(x^{\prime}\right)$ is a win for $\beta$ if $x \in A_{2}-A_{1}$,

(c) the mapping $x \rightarrow x^{\prime}$ is Borel measurable, and

(d) for every ordered pair $\left(A_{1}, A_{2}\right)$ of nonempty analytic subsets of $X$, there is $x \in X$ such that $\left(A_{1}, A_{2}\right)$ is associated with $x$.

We shall say that " $x$ codes $\left(A_{1}, A_{2}\right)$ " in case $\left(A_{1}, A_{2}\right)$ is associated with $x$.

3. We are now ready to state our example. Let $D=X-\pi\left(B_{1}\right)$, and let $H=X-\pi\left(B_{2}\right)$. In other words, $D$ is the set of $x \in X$ such that $G(x)$ is a win for $\beta$, while $H$ is the set of $x$ 's such that $G(x)$ is a win for $\alpha_{0}$ We claim that $D, H$ are disjoint coanalytic sets which are not Borel separable.

From (i) it follows that $D$ and $H$ are coanalytic. (ii) implies that $D \cap$ $H=\varnothing$. Next we note that $D, H$ are nonempty. To see this, let $x$ be the singleton set whose only member is the ordered pair $(1,2)$. Plainly, $G(x)$ is a win for $\beta$, so that $x \in D$. A similar argument shows that $H \neq \varnothing$.

Now assume by way of contradiction that there is a Borel set $E \subseteq X$ such that $D \subseteq E$ and $E \cap H=\varnothing$. Plainly, $E$ and $X-E$ are both nonempty. Let $W=\left\{x \in X: x^{\prime} \in E\right\}$. By (iii)(c), $W$ is Borel. We now assert that both $W$ and $X-W$ are nonempty. To see, for instance, that $W \neq \varnothing$, choose $x_{0} \in X$ and a nonempty analytic set $C \subseteq X$ such that $x_{0}$ codes $(C, X)$ and $x_{0} \notin C$ (we can do this; for, if not, then for each $z \in X, z$ codes $(\{z\}, X)$, so that there are no codes left for the other pairs). Since $x_{0} \in X-C$, it follows from (iii)(b) that $G\left(x_{0}^{\prime}\right)$ is a win for $\beta$, so $x_{0}^{\prime} \in D$ and hence, $x_{0}$ $\epsilon W$. One shows $X-W \neq \varnothing$ similarly.

Consequently, from (iii)(d), there is $x^{*} \in X$ such that $x^{*} \operatorname{codes}(W, X-$ W.). We now have: $x^{*^{\prime}} \in E \rightarrow x^{*} \in W \rightarrow G\left(x^{*^{\prime}}\right)$ is a win for $a \rightarrow x^{*^{\prime}} \in H \rightarrow x^{*^{\prime}} \notin E$; and also, 
$x^{*^{\prime}} \notin E \rightarrow x^{*} \in X-W \rightarrow G\left(x^{*^{\prime}}\right)$ is a win for $\beta \rightarrow x^{*^{\prime}} \in D \rightarrow x^{*^{\prime}} \in E$, so that $x^{*^{\prime}} \in E \leftrightarrow x^{*^{\prime}} \notin E$, which yields the desired contradiction.

4. Finally, by using an argument due to Novikov [4, p. 25], one can deduce from the fact that $D$ and $H$ are not Borel separable that the set $B=B_{1}$ $\cup B_{2}$ does not admit a Borel uniformization. Indeed, suppose that $S$ is a Borel uniformization of $B$. Let $T=\pi\left(S-B_{1}\right)$. Since $\pi$ is continuous and one-one on $S, T$ is Borel [2, p. 487]. Now verify that $D \subseteq T$ and $T \cap H=\varnothing$, which contradicts the fact that $D$ and $H$ are not Borel separable.

\section{BIBLIOGRAPHY}

1. D. Blackwell, A Borel set not containing a graph, Ann. Math. Statist. 39 (1968), 1345-1347. MR 37 \#5025.

2. K. Kuratowski, Topology. Vol. 1, new ed., rev. and augmented, PWN, Warsaw; Academic Press, New York, 1966. MR 36 \#840.

3. N. N. Luzin, Leçons sur les ensembles analytiques et leurs applications, Gauthier-Villars, Paris, 1930.

4. P. Novikov, Sur les fonctions implicites mesurables B, Fund. Math. 17 (1931), 8-25.

5. W. Sierpiński, Sur deux complémentaries analytiques non séparables $B, \mathrm{~F}$ und. Math. 17 (1931), 296-297.

DEPARTMENT OF STATISTICS, UNIVERSITY OF CALIFORNIA, BERKELEY, CALIFORNIA 94720

Current address: Indian Statistical Institute, Calcutta, India 